\title{
What is Ensemble Librarianship?
}

\author{
Erika Kirsch \\ McGill University
}

Within the world of the information profession, music librarianship is a highly specialized area. In addition to dealing with books and journals, music librarians work with sources unique to the discipline: scores, numerous recording formats, discographies, repertoire guides and thematic catalogs, to name a few. But what about ensemble librarianship-the mysterious cousin of music librarianship-a subset of this specialization? Where do ensemble librarians work, and what do they do?

Ensemble librarians are found in professional performing organizations of many types: orchestras, opera companies, and ballet companies, as well as in military branches. There are those of us who work in the music departments of universities, colleges and conservatories. Finally, ensemble librarians are employed by the rental departments of many publishers and publishers' agents.

In some shape or form, ensemble librarians all perform the following functions: acquiring, cataloguing, processing, circulating and maintaining all the separate scores and parts for large and small ensembles - orchestra, wind symphony and band, contemporary music ensemble, jazz ensemble, chamber ensemble, opera orchestra, choir, etc. In other words, they manage all the sheet music those people crowded onto the stage and in the orchestra pit are using to create the magic of live concerts. Ensemble librarians collaborate with conductors to make sure that important markings are put into the parts, including errata markings, rehearsal systems and cuts. Those of us working in the university setting have the added challenge of circulating parts to a constantly rotating list of students in different ensembles. Ensemble librarians have incredible collective knowledge about performing editions and how to find them, and numerous creative techniques for binding the pages of parts and scores together. They have a special understanding of copyright laws related to the use of sheet music for public performances, recordings, dramatized performances, and "-casts"-webcasts, broadcasts, podcasts. Finally, ensemble librarians are adept at handling the complexities of renting music from agents and publishers and are well-versed in the varied and sometimes obscure resources used for finding large ensemble music.

University ensemble librarians have the unique opportunity to work with composers in the earlier stages of their development. They are on hand to help answer young composers' questions on creating their first sets of parts and scores and, in so doing, they facilitate the first live performances of their works. An added bonus is that this helps budding composers make a good

(C) 2010 The author and the Canadian Association of Music Libraries, Archives and Documentation Centres / L'auteur et l'Association canadienne des bibliothèques, archives et centres de documentation musicaux 
impression on fellow students who are creating these premieres. Later in their careers, composers might again encounter ensemble librarians who, as part of a professional performing organization, are involved in working out the details of commissioning agreements.

The professional organization that ties ensemble librarians together is the Major Orchestra Librarians' Association (MOLA). Founded in 1983, it has grown into an international association of more than 250 organizations, represented by more than 400 ensemble librarians. The skills and knowledge of ensemble librarianship have not traditionally been taught inside music or library science degree programs. Instead, mentoring and informal one-on-one teaching/learning have been the norm, with the annual conference enabling us to gather together, share information and resources, and discuss the most current and relevant issues we face.

However, MOLA's Education Committee recently made a grand step in this area by planning and holding its first regional workshop, bringing the knowledge of our experienced members to those less experienced - a sort of "house call"! The workshop was held on October 9, 2010, on the Evanston campus of Northwestern University (NU) in the Chicagoland area. Co-sponsored by NU's Bienen School of Music, the workshop attracted nearly 40 attendees. The presenters were Mark Swanson, Carole Keller and Peter Conover (all 3 from the Chicago Symphony Orchestra), Wendy Skoczen (Chicago Lyric Opera), Michael Shelton (Grant Park Music Festival) and Pat McGinn (Milwaukee Symphony Orchestra). Topics presented were: Materials and Resources; Customer Service and Communication; Music Preparation, Repair and Binding; Acquisitions and Rental; Research Methods; and Copyright.

The presentations were excellent, and many even included informative handouts. Most ensemble librarians are not used to speaking in front of large groups-it is definitely not a regular part of the job for most of us!-but you would never know it from this crew; they seemed completely at ease discussing their various topics in front of the attendees. It was enlightening to hear Pat McGinn and Peter Conover speak about communication and elaborate on the reality that our unique positions mean we are constantly feeling our way delicately and diplomatically through many different types of situations. Carole Keller and Wendy Skoczen discussed acquisitions and rental issues expertly, providing an excellent bridge between the discussions of research and copyright. The information on copyright shared by Wendy Skoczen and Michael Shelton was an eye-opener for many. This is a murky and difficult area in ensemble librarianship, but an important subject to broach, especially for those who don't have many years of experience.

The presenters took questions throughout the sessions, and there was a $\mathrm{Q} \& \mathrm{~A}$ session at the end; the attendees were quite keen and asked many relevant questions. Everyone in attendance, regardless of their level of experience, went away having learned something new. I, for one, learned from the presentation on resources that there is a forthcoming volume on finding operarelated excerpts being prepared by John Yaffé and David Daniels. I heard many attendees talking about how helpful it was to see all the tools of the trade displayed: hinging tape, binding supplies and correction tape, among others. They also appreciated the demonstrations of part binding, and valued learning about the various resources for finding music for performing ensembles.

The number of attendees and their enthusiasm was an encouraging indication that there are many people interested in learning about our specialized field. Some people came from university 
library science programs, some from local performing ensembles, and still others were students at college/university music degree programs in the Chicagoland area. People learned about the workshop from the MOLA website, from emails and snail mail sent by the committee, or from the various listservs on which information was posted.

In closing, I will mention a couple of valuable resources. To learn more about ensemble librarianship you can visit the MOLA website: http://www.mola-inc.org. The site contains links to many useful resources, including music preparation guidelines for composers, information on searching for elusive publications, and a listing of both summer jobs and internships/fellowships/assistantships in the field of ensemble librarianship. In addition, Russ Girsberger, who is the Ensemble Librarian at the Juilliard School, has authored a fine book on ensemble librarianship, A Manual for the Performance Library (Scarecrow Press, 2006).

If you have questions about the field, you can contact any one of the MOLA members through the email addresses provided on the MOLA website.

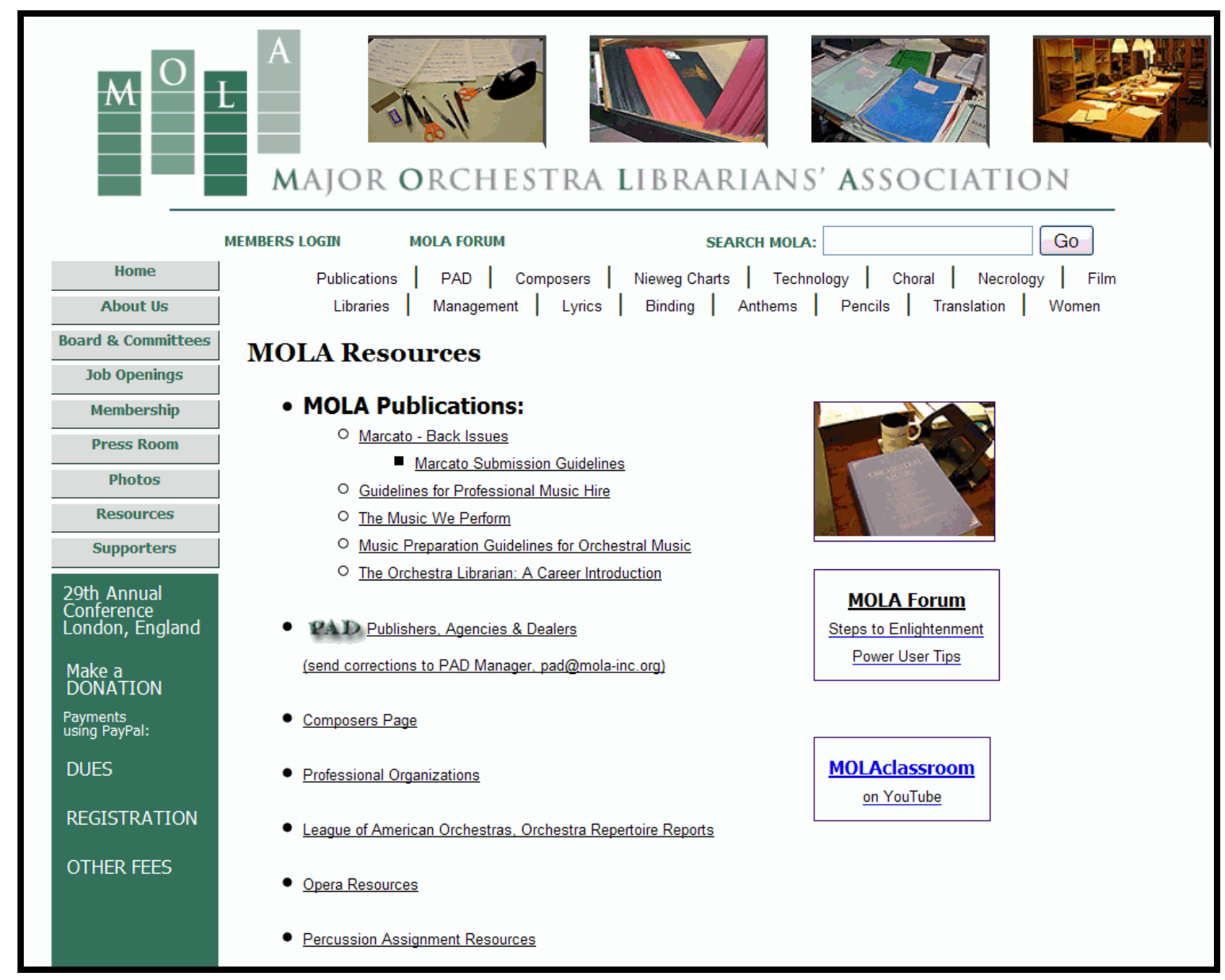

http://www.mola-inc.org 